\title{
Diseño de un curso MOOC como estrategia para fortalecer las sinergias comunitarias en las zonas rurales
}

Design of a MOOC course as a strategy to strengthen community synergies in rural areas

Mayra Roxana Solarte Montoya ${ }^{1}$

José Fernando Grass Ramírez ${ }^{2}$ ilimitado, distribución y reproducción en cualquier medio, siempre que el autor original y la fuente se acreditan.

\section{RESUMEN}

Los territorios rurales en Colombia se caracterizan por ser escenarios interculturales, este aspecto plantea retos a la formación profesional tradicional, ofreciendo alternativas como el proyecto MOOCMenTes, en el cual se aprovechan espacios virtuales y el tránsito a la Educación Superior desde contextos rurales y diversos. Este artículo evidencia una estrategia para fortalecer las sinergias comunitarias en las zonas rurales, el diseño del curso MOOC "Construcción colectiva de mercados agroecológicos y solidarios", utilizando la herramienta MOOC Canvas para el diseño del curso, y su validación a través de una herramienta metodológica de Investigación-Acción-Participativa (IAP). En esta experiencia se logró afianzar una modalidad de formación como son los MOOCs, con una temática pertinente hacia los territorios rurales, como dinamizadora de la educación rural.

PALABRAS CLAVE: curso en línea, educación rural, mercados agroecológicos, MOOC, sinergias comunitarias.

\section{ABSTRACT}

Rural territories in Colombia are characterized by intercultural diversity, this aspect raised a lot of challenges in the vocational training that are traditional and alternatives such as the MOOCMenTes project, this one have virtual spaces that are leverage that they have been proliferated because it make an expansion of connectivity in the country and in the transition to get Higher Education from urban to rural contexts. This article demonstrates a strategy to strengthen the community synergies in rural areas, the design of the MOOC course "Collective construction of agroecological and solidarity markets", using the MOOC Canvas tool for the design of the course, and its validation through a methodological tool of Participatory-Action-Research. In this experience, it was possible to consolidated a training modality such as the MOOCs, with a relevant theme towards rural territories, as a catalyst for the rural education.

KEYWORDS: online course, rural education, agroecological markets, MOOC, community synergies.

\footnotetext{
${ }^{1}$ Magíster en Agronegocios. Ingeniera Agroindustrial. Investigadora, Grupo de investigación para el Desarrollo Rural TULL, Universidad del Cauca. Colombia. mayrasolarte@unicauca.edu.co

${ }^{2}$ Doctor en Problemas Económico Agroindustriales. Ingeniero Químico. Profesor titular, Facultad de Ciencias Agrarias, Departamento de Agroindustria. Grupo de investigación para el Desarrollo Rural TULL - Universidad del Cauca. Popayán, Colombia.jfgrass@unicauca.edu.co
} 


\section{INTRODUCCIÓN}

En este artículo se plantea el diseño del contenido de un curso MOOC, en su acrónimo en inglés Massive Online Open Courses, es decir, cursos abiertos masivos y en línea, como alternativa educativa para espacios rurales y como estrategias para fortalecer las sinergias comunitarias en los territorios, el cual se gesta en el Proyecto MOOCMenTES, para dinamizar y articular a los actores del sector rural en un espacio donde se tejen las realidades de los contextos rurales y urbanos. Como premisa, MOOCMenTes es un proyecto de la Universidad del Cauca, co-financiado en el marco de alianzas rurales por el Ministerio de Educación Nacional de Colombia, que tiene como objetivo la formación profesional rural, en espacios virtuales y el tránsito a la Educación Superior hacía contextos rurales y diversos (Unicauca, 2018).

La educación, en este caso, la rural, debe proveer a los jóvenes de un conjunto de competencias para comprender la vida social y económica de sus territorios, a través de aprendizajes basados en proyectos productivos y sinergias comunitarias de diverso tipo, según las particularidades del entorno donde posiblemente ocurrirá su desempeño laboral. Para el Ministerio de Educación Nacional de Colombia (MinEducacón, 2012), la vinculación de personas que viven en territorios rurales a nuevas tecnologías y medios de comunicación, no son reconocidos como factores que causan aislamiento, sino que por el contrario, facilitan el desarrollo rural con enfoque territorial, con la premura de que es necesaria la participación activa de los actores locales para garantizar un proceso endógeno de cambio. La pertinencia en la educación rural, hace referencia a una educación útil para la vida, que les permita a los jóvenes rurales una conexión de las relaciones sociales en sus veredas o corregimientos, con el mundo económico, que los prepare en competencias para el emprendimiento, la responsabilidad personal, y les facilite el uso de los desarrollos tecnológicos en el campo de la información y la comunicación. Actualmente, son escasos los espacios virtuales que fomentan la vocación rural comunitaria en los territorios, además, existen dificultades en el momento de transferir el conocimiento, barreras que se interponen en el intercambio de saberes, sobre todo en la inclusión de los jóvenes a las dinámicas rurales que fortalezcan el relevo generacional. En ese sentido, en la identificación de las necesidades para la educación rural, los actores de estos espacios destacan la necesidad de proponer alternativas para mitigar los reducidos ingresos, fomentar la economía y agricultura familiar, la falta de precios justos a los productos, estimular agregación de valor a la producción, vincularse a nuevos circuitos comerciales y la necesidad de fortalecer las sinergias comunitarias entre las organizaciones sociales de base que ayuden a incentivar la Soberanía Alimentaria y los Circuitos Cortos de Comercialización. Es por ello que surge la pregunta de investigación, ¿Cómo establecer relaciones entre la educación virtual que fortalezca la construcción colectiva de procesos locales en los territorios rurales?

En ese sentido, se diseñó el contenido del curso MOOC "Construcción colectiva de mercados agroecológicos y solidarios", como una oportunidad de hacer uso de las tecnologías interactivas, fomentar el desarrollo rural, fortalecer los Circuitos Cortos de Comercialización, disminuir las brechas entre productores rurales y urbanos con el consumidor, y promover la conectividad rural. Con este curso MOOC se busca que los aprendices en formación, fortalezcan sus competencias para llevar a cabo mercados agroecológicos y solidarios locales en su territorio, aunando esfuerzos con las organizaciones sociales rurales y colectivos urbanos, en búsqueda de la Soberanía Alimentaria, la solidaridad entre los participantes, y la comercialización de sus productos frescos, transformados naturales, semillas nativas y criollas, artesanías locales, manualidades sustentables y reuso de materiales. El curso diseñado, se pretende ofertar en la Plataforma Selene de la Universidad del Cauca, en Colombia.

El objetivo general de este artículo, es evidenciar como estrategia para fortalecer las sinergias comunitarias en las zonas rurales, el diseño del curso MOOC "Construcción colectiva de mercados agroecológicos y solidarios". Del mismo modo, los objetivos específicos son: 1) Utilizar la herramienta MOOC Canvas para el diseño del curso; 2) Validar el MOOC Canvas a través de una herramienta participativa.

\section{Metodología}

En esta sección, se plantea el enfoque metodológico para llevar a cabo los objetivo planteados en este artículo, considerando en un primer momento, la herramienta que se utilizó como estructura para el diseño del curso MOOC y posteriormente, en un segundo momento, la validación del curso a través 
de la Investigación-Acción-Participativa (IAP), con los actores rurales a quienes estará dirigido el curso.

\section{MOOC Canvas}

El MOOC Canvas como herramienta metodológica para diseñar y describir los elementos que componen el curso, interrelaciona las temáticas de logística, tecnología y pedagogía, necesarias para la elaboración de la estructura del MOOC (AlarioHoyos, et al, 2013), con las premisas que los educadores necesitan pensar también durante el diseño del curso, en la temática financiera (AlarioHoyos, et al, 2014). Esta herramienta, permitió obtener una visión general de los recursos disponibles para la puesta en marcha del curso, y posteriormente, realizar los ajustes pertinentes con el equipo de trabajo. Para el MOOC Canvas, según Alirio-Hoyos et al (2013), existen once (11) elementos que están organizados en dos categorías: recursos disponibles y diseño decisiones. Los recursos disponibles son aquellos recursos clave con los que profesores disponen a la hora de diseñar el curso, paralelo a ello, los recursos deben ser acordados a nivel institucional, como por ejemplo, las características que ofrece la plataforma, en este caso Selene. Para mencionar estos recursos, se mencionan a continuación: 1) Recursos Humanos; 2) Recursos Intelectuales; 3) Equipamiento; 4) Plataforma. Las decisiones de diseño, se ven afectadas por los recursos disponibles y sus elementos se interrelacionan y son dependientes unas de otras. Éstas son: 5) Descripción general; 6) Estudiantes a los que va dirigido; 7) Aproximaciones Pedagógicas; 8) Objetivos y Competencias; 9) Contenidos de Aprendizaje; 10) Actividades de Evaluación; y, 11) Tecnologías Complementarias.

Para complementar, en el MOOC Canvas no se consideran los recursos financieros como un tema separado, en este recurso puede intercambiarse por recursos humanos, recursos intelectuales, compra de equipos y pagar una tarifa por el uso de plataformas (Alario-Hoyos, et al, 2014).

\section{Investigación-Acción-Participativa (IAP)}

El modelo que se propone para la evaluación y el Para la validación de este proceso, se buscó emprender acciones e iniciativas comunitarias soportadas en procesos participativos como la Investigación-Acción-Participativa (IAP), mediante el diálogo de saberes con los actores objetivo, quienes impulsan y materializan las acciones proyectadas a través de la educación rural virtual, con el curso MOOC. Para Alberich (2008), la IAP (Investigación-Acción-Participativa) como metodología, es un proceso de investigación que incluye las negociaciones y participación plural en procesos complejos de diagnóstico, análisis y acción social. Según, Basagoiti, et al (2001), la IAP es una metodología de investigación y también, un proceso de intervención social, donde se propone el análisis de la realidad del contexto, como una forma de conocimiento y sensibilización de la población objeto de estudio activo y protagonista de un proyecto de desarrollo y transformación de su entorno.

\section{Resultados}

Los resultados arrojados en esta sección, consisten básicamente en presentar el contenido diligenciado en el MOOC Canvas para el curso "Construcción colectiva de mercados agroecológicos y solidarios", y enseguida se plasma el análisis e importancia de la validación del diseño del curso con los actores objeto.

\section{Diseño del MOOC Canvas}

En el curso diseñado se abordan los elementos claves para la construcción colectiva de mercados agroecológicos y solidarios. Los principales aspectos se capturan en el MOOC Canvas, donde se incluye la descripción de los recursos disponibles y las principales decisiones de diseño. El equipo de diseño del curso, estuvo conformado por tres (3) profesores, siendo la primera experiencia para cada uno de ellos en el diseño de MOOC. Para conseguir la versión definitiva del MOOC Canvas del curso, se elaboraron diez (10) versiones, las cuales se iban afinando una vez se socializaban a expertos invitados al proyecto MOOCMenTes, a los asesores del proyecto y entre los integrantes del equipo del diseño del curso MOOC.

En efecto, se diligenciaron los once (11) elementos del MOOC Canvas, que componen los recursos disponibles y las decisiones de diseño. Los recursos disponibles, que son cuatro (4), se describen a continuación:

\subsection{Recursos Humanos:}


profesores y/o expertos en Desarrollo Rural y Telemática; Profesionales o técnicos en Comunicación Social, multimedia, audiovisual; Administrador de la plataforma Selene Unicauca.

\subsection{Recursos Intelectuales:}

Cartillas informativas, artículos y videos informativos.

\subsection{Equipamiento:}

Recursos hardware (estudio de grabación, cámara fotográfica, cámara de video, micrófono, computador portátil) y recursos software (licencia del Sistema Operativo, herramienta de libre distribución que permita crear tutoriales en video y presentaciones vía screencast - grabación digital de la salida por pantalla del computador, reproductor y editor de videos de libre distribución).

\subsection{Plataforma:}

La Plataforma Selene de la Universidad del Cauca. Los tipos de formatos soportados por la plataforma son textos (PDF, txt), Imágenes (.gif y .jpg), videos (mp4), audios (mp3, wma) y otros (html). Entre los tipos de actividades de evaluación de evaluación soportados por la plataforma, se encuentra los test de respuesta múltiple, menú desplegable y evaluación entre pares. Por último, las herramientas sociales soportadas por la plataforma, son los foros de discusión y las herramientas de preguntas y respuestas.

Las decisiones de diseño, encaminadas a describir los elementos de mayor importancia en el MOOC Canvas, se relacionan seguidamente:

\subsection{Descripción general:}

El nombre del curso es "Construcción colectiva de mercados agroecológicos y solidarios". El curso tendrá una duración de cuatro (4) semanas. Las áreas de conocimiento abordadas son: Desarrollo Rural, Soberanía Alimentaria, Agroecología, Economía Solidaria, Sistemas Agroalimentarios Localizados.

\subsection{Estudiantes a los que va dirigido:}

Inicialmente a estudiantes colombianos, con un nivel educativo a partir de la Educación Media, con conocimientos básicos en el uso de computadores (uso de programas de ofimática y navegación en internet). En el caso del sector profesional, irá dirigido a personas involucradas en el sector rural como extensionistas rurales, profesores de Educación Media, estudiantes de Educación
Media, líderes sociales y emprendedores rurales y urbanos. La motivación para acceder a este curso, está encaminada a que los aprendices fortalezcan las competencias para organizar comunitariamente un mercado agroecológico y solidario, y reconozcan prácticas de intercambio comercial y solidario.

\subsection{Aproximaciones Pedagógicas:}

El curso MOOC considera las siguientes estrategias y recursos didácticos: capacitación en el uso de la plataforma Selene Unicauca para cursar el MOOC, presentaciones trasmitidas a través de videos, y aprendizajes basados en proyectos productivos y en sinergias comunitarias, estudios de caso y soluciones prácticas a problemas.

\subsection{Objetivos y Competencias:}

El objetivo general del curso MOOC es conocer los elementos para la construcción colectiva de mercados agroecológicos y solidarios. A su vez, los objetivos específicos son:

1) comprender los conceptos, antecedentes y reflexiones como base en la construcción colectiva del mercado;

2) Conocer las dinámicas logísticas y organizativas del mercado;

3) Reconocer prácticas de intercambio comercial y solidario.

La competencia que adquiere los aprendices una vez aprueben el curso MOOC, es asociar los elementos claves para construir comunitariamente un mercado agroecológico y solidario en un contexto intercultural.

\subsection{Contenidos de Aprendizaje:}

La estructura del MOOC está compuesta por 4 secciones, cada sección corresponde a una semana de duración.

La sección 1, corresponde a la introducción del estudiante al curso, un conjunto de actividades de ambientación, definición y antecedentes de la temática. La sección 2, hace referencia básicamente a los conceptos y reflexiones a tener en cuenta en la construcción comunitaria de un mercado agroecológico y solidario. La sección 3, corresponde a conocer los roles de los actores, las dinámicas de la logística y organización comunitaria de cómo se construye colectivamente un mercado agroecológico y solidario. Por último, en la sección 4 , se presentan algunas prácticas de intercambio comercial y solidario.

\subsection{Actividades de Evaluación:}

Se componen de dos evaluaciones, la formativa y la 
Tabla 1.Contenidos de aprendizaje del curso MOOC

\begin{tabular}{|c|c|c|c|}
\hline \multicolumn{4}{|c|}{ Estructura de los contenidos } \\
\hline Sección & Subsección & Unidad & Formatos de los contenidos \\
\hline \multirow{5}{*}{$\begin{array}{l}\text { Sección 1: } \\
\text { Introducción- } \\
\text { Ambientación }\end{array}$} & & 1.1.1 Inducción al curso & $\begin{array}{l}\text { Video de presentación } \\
\text { (Objetivos de aprendizaje, } \\
\text { presentación de docentes) }\end{array}$ \\
\hline & 1.2 Uso de la & $\begin{array}{l}\text { 1.2.1 Uso y exploración de los recursos del sitio en } \\
\text { la Plataforma Selene Unicauca }\end{array}$ & $\begin{array}{l}\text { - Video tutorial genérico } \\
\text { - Textos }\end{array}$ \\
\hline & \multirow[t]{2}{*}{ 1.3 Definicióny } & $\begin{array}{l}\text { 1.3.1 ¿Qué son los mercados agroecológicos y soli- } \\
\text { darios? }\end{array}$ & $\begin{array}{l}\text { Concepto por parte de los } \\
\text { docentes } \\
\text { - Conceptos por parte de los } \\
\text { invitados (líderes comunitarios) } \\
\text { - Página web }\end{array}$ \\
\hline & & 1.3.2 Tipos de mercados comunitarios & - Textos \\
\hline & & 1.4.1 Presentación personal de los aprendices & - Textos \\
\hline \multirow[t]{2}{*}{$\begin{array}{l}\text { Sección 2: } \\
\text { Conceptos y } \\
\text { reflexiones }\end{array}$} & 1.1Conceptos & $\begin{array}{ll}\text { 1.1.1 } & \text { Soberanía Alimentaria } \\
\text { 1.1.2 } & \text { Agroecología } \\
\text { 1.1.3 } & \text { Economía Solidaria } \\
\text { 1.1.4 } & \text { Circuitos Cortos de Comercialización } \\
1.1 .5 & \text { Sistemas Agroalimentarios Localizados } \\
\text { 1.1.6 } & \begin{array}{l}\text { Relación de conceptos con los mercados } \\
\text { agroecológicos y solidarios }\end{array} \\
\end{array}$ & $\begin{array}{l}\text { Conceptos por parte de los } \\
\text { docentes invitados } \\
\text { Ejemplos de los conceptos por } \\
\text { parte de los docentes }\end{array}$ \\
\hline & $\begin{array}{l}2.2 \\
\text { Reflexiones }\end{array}$ & $\begin{array}{l}\text { 2.2.1 ¿Cómo se producen los alimentos que } \\
\text { encontramos en un mercado agroecológico local y de } \\
\text { dónde provienen? } \\
\text { 2.2.2 La agroecología como apuesta política de los } \\
\text { movimientos sociales. } \\
\text { 2.2.3 Seguridad Alimentaria, Soberanía Alimentaria, } \\
\text { Autonomía Alimentaria: ¿se diferencian o se } \\
\text { complementan? }\end{array}$ & $\begin{array}{l}\text { Transparencias } \\
\text { Textos }\end{array}$ \\
\hline \multirow{5}{*}{$\begin{array}{l}\text { Sección 3: } \\
\text { Logística y } \\
\text { organización } \\
\text { comunitaria }\end{array}$} & & 3.1.1 Roles de los productores locales & $\begin{array}{l}\text { - Notas } 360^{\circ} \\
\text { - Transparencias }\end{array}$ \\
\hline & & 1.1.2 Roles de los consumidores & $\begin{array}{l}\text { - Video selfie } \\
\text { - Página web }\end{array}$ \\
\hline & \multirow{3}{*}{$\begin{array}{l}3.2 \text { Paso a } \\
\text { paso de las } \\
\text { dinámicas } \\
\text { logísticas y } \\
\text { organizativas }\end{array}$} & 3.2.1 Antes del mercado & \multirow{3}{*}{$\begin{array}{l}\text { Video con voz en off } \\
\text { - Texto }\end{array}$} \\
\hline & & 1.1.2 Durante el mercado & \\
\hline & & 1.1.3 Después del mercado & \\
\hline \multirow{2}{*}{$\begin{array}{l}\text { Sección 4: } \\
\text { Prácticas de } \\
\text { intercambio } \\
\text { comercial y } \\
\text { solidario }\end{array}$} & \multirow{2}{*}{$\begin{array}{l}4.1 \\
\text { Estrategias de } \\
\text { diversificación } \\
\text { del ingreso }\end{array}$} & 4.1.1 Trueque & $\begin{array}{l}\text { - Mini-documentales con cada } \\
\text { unidad }\end{array}$ \\
\hline & & 1.1.2 Gastronomía local & $\begin{array}{l}\text { - Textos } \\
\cdot \text { Páginas web } \\
\cdot \text { Transparencias }\end{array}$ \\
\hline
\end{tabular}


sumativa. Como evaluación formativa, se planteó realizar uno o dos tipos de exámenes para cada una de las secciones: test de respuesta múltiple, frases incompletas, enunciados falso o verdadero y juegos lúdicos como el ahorcado. Como evaluación sumativa, se planteó realizar uno o dos tipos de exámenes para cada una de las secciones: video de presentación personal de los aprendices, test de respuesta múltiple, menú desplegable, videos de experiencias reales, evaluación de pares a videos de experiencias reales y exposición de estudios de caso reales.

\subsection{Tecnologías Complementarias:}

En cuanto a los contenidos de plataforma externos se hará uso de las siguiente plataformas web externas: Agronet: http://www.agronet.gov. $\mathrm{co} /$, y la Plataforma de conocimientos sobre agricultura familiar http://www.fao.org/familyfarming/countries/col/es/ de la Organización de las Naciones Unidas para la Alimentación y la Agricultura (FAO). Por último, para promover la comunicación y discusión entre los estudiantes, se hará uso de redes sociales como Facebook.

\section{Herramienta participativa para validación del MOOC Canvas}

La herramienta participativa empleada para validar el contenido del curso MOOC con los actores rurales, se diseñó con el equipo de trabajo dirigido principalmente a jóvenes provenientes de zonas rurales de los municipios de Cajibío, Caldono, El Tambo, Popayán, Puracé, Santander de Quilichao, Sotará, Suárez y Timbío, del Departamento del Cauca, Colombia. En la jornada de validación del curso, inicialmente se socializó a los trece (13) participantes, los objetivos del proyecto MOOCMenTes, y seguidamente, el contenido del MOOC Canvas a través de diapositivas. Posteriormente, se aplicó la herramienta participativa de validación del curso.
El relevo generacional es una de las estrategias a fortalecer en la Educación Rural hacia el tránsito a la Educación Superior, es por ello, que los líderes sociales mostraron su interés en que los jóvenes de sus comunidades, asistieran al taller participativo que el proyecto MOOCMenTes realizó en el Centro de Estudios Vegetales y Diversidad Asociada La Rejoya de la Universidad del Cauca. Los jóvenes hacen parte de las organizaciones sociales campesinas, indígenas y afros del Departamento del Cauca, entre las que se encuentran: Institución Educativa Técnica Agrícola de Suárez - Municipio de Suárez, Asociación Nacional de Usuarios Campesinos Nodo Cauca - Municipios de Santander de Quilichao, Movimiento Campesino de Cajibío Municipio de Cajibío, Organización Nuevo Futuro - Municipios de El Tambo y Timbío, Asociación Ambientalista de Popayán (ASOCAMPO) Municipio de Popayán, y el Asentamiento Indígena de Sachacoco - Municipio de Sotará; la edad de los jóvenes estuvo comprendida entre los 14 y 27 años.

\subsection{Primer momento de la herramienta de validación.}

Estuvo orientada a responder participativamente, cuatro (4) preguntas a manera individual, donde los participantes utilizaron el material suministrado como papeles de colores, marcadores, lápices y cinta adhesiva. Las preguntas orientadoras fueron las siguientes: a) ¿Qué conocimientos vas a adquirir en ese MOOC?; b) El tema de curso, ¿es pertinente a las necesidades de tu comunidad?; c) ¿Quiénes estarían interesados en hacer el curso en tu comunidad?; d) ¿Tienen acceso a internet en tu comunidad? ¿Dónde? ¿O cuál es el lugar más cercano donde podrían tener acceso a internet?

Los ejes de las secciones, se identificaron una vez se clasificaron las respuestas de los participantes. A continuación, se muestran los resultados de la primera pregunta orientadora:

Tabla 2Respuestas de la pregunta orientadora, ¿Qué conocimientos vas a adquirir en este MOOC?

\begin{tabular}{|c|l|}
\hline Ejes de las secciones & Respuestas \\
\hline $\begin{array}{c}\text { Definición y antecedentes } \\
\text { de los mercados } \\
\text { agroecológicos y } \\
\text { solidarios }\end{array}$ & - Conocer más sobre los mercados agroecológicos \\
\hline
\end{tabular}




\begin{tabular}{|c|c|}
\hline Conceptos y reflexiones & $\begin{array}{l}\text { - Preservar las semillas nativas } \\
\text { - Ser más consciente sobre los productos que llevamos a la mesa } \\
\text { - Diferenciar productos propios y los no propios } \\
\text { - Conocer la conceptualización, acerca de la producción más limpia } \\
\text { - Poder adquirir un conocimiento más avanzado de todo lo que es el campo y su } \\
\text { producción en conjunto con la soberanía alimentaria } \\
\text { - Conceptos de seguridad alimentaria, soberanía alimentaria, fuentes adicionales de } \\
\text { ingreso } \\
\text { - Conocer nueva terminología }\end{array}$ \\
\hline Logística & $\begin{array}{l}\text { - Cómo debo organizarme antes del desarrollo del mercado } \\
\text { - Realizar una planificación de las actividades } \\
\text { - Poder realizar mercados y estrategias para poder ayudar y salvar algunos productos }\end{array}$ \\
\hline Organización comunitaria & $\begin{array}{l}\text { - Generar más consciencia y sentido de pertenencia } \\
\text { - Aumentar los beneficios para las organizaciones } \\
\text { - Valorar los productos generados en nuestros territorios } \\
\text { - Poder conocer los productos sembrados en las fincas, en los mercados } \\
\text { - Trabajar colectivamente } \\
\text { - Tener una visión más amplia referente a la implementación de los mercados } \\
\text { comunitarios }\end{array}$ \\
\hline $\begin{array}{l}\text { Prácticas de intercambio } \\
\text { comercial y solidario }\end{array}$ & $\begin{array}{l}\text { - Generar valor agregado } \\
\text { - Conocer todos los productos y generar valor agregado } \\
\text { - Dar a conocer mis productos } \\
\text { - Cultivar de forma sostenible }\end{array}$ \\
\hline
\end{tabular}

Los ejes de las secciones, se identificaron una vez se clasificaron las respuestas de los participantes. A continuación, se muestran los resultados de la primera pregunta orientadora: En la segunda pregunta orientadora, también se realizó la clasificación de las respuestas, esta vez, organizándolas respecto a los conceptos abordados en el contenido del curso. Los participantes evidenciaron en un $100 \%$ que las temáticas del curso son pertinentes a las necesidades en sus comunidades, respecto a la Soberanía Alimentaria, la Agroecología, la Economía Solidaria, los Circuitos Cortos de Comercialización y los Sistemas Agroalimentarios Localizados.

Tabla 3. Respuestas de la pregunta orientadora, el tema de curso, ¿es pertinente a las necesidades de tu comunidad?

\begin{tabular}{|c|c|}
\hline Conceptos & Respuestas \\
\hline Soberanía Alimentaria & $\begin{array}{l}\text { - Sí, porque generamos el espacio para se haga viral, que los productos son sanos y } \\
\text { así mejorar la calidad de vida de cada uno de los productores. } \\
\text { - Enseñar a consumir lo propio. } \\
\text { - Sí, porque no ayuda a rescatar saberes ancestrales y costumbres que se han } \\
\text { perdido en el transcurso de los años } \\
\text { - Sí, porque en nuestra asociación manejamos un proyecto de seguridad y soberanía } \\
\text { alimentaria, bajo el establecimiento de huertas caseras con } 50 \text { familias }\end{array}$ \\
\hline Agroecología & $\begin{array}{l}\text { - Sí, primero porque es incluyente y segundo, porque maneja una metodología apta } \\
\text { para cualquier tipo de persona } \\
\text { - Sí, porque en nuestra comunidad se produce gran diversidad de alimentos/ } \\
\text { productos } \\
\text { - Sí, porque mi comunidad es productora del campo y con este curso } \\
\text { complementaría las estrategias para hacer un buen mercado de sus productos o } \\
\text { cosechas }\end{array}$ \\
\hline Economía Solidaria & $\begin{array}{l}\text { - Sí, porque al socializar el curso a la comunidad, ayudaría a su mejoramiento las } \\
\text { necesidad del agro y su ámbito comunitario y social del territorio } \\
\text { - Sí, debido a que el sector rural es muy escasa la comunicación de este tipo y la } \\
\text { gran mayoría ignora muchas estrategias de mercados } \\
\text { - Si, porque de esta forma está fortaleciendo tanto en los mercados y a las } \\
\text { organizaciones }\end{array}$ \\
\hline
\end{tabular}




\begin{tabular}{|c|l|}
\hline $\begin{array}{c}\text { Circuitos Cortos de } \\
\text { Comercialización }\end{array}$ & - Sí, porque así ayudamos a que los campesinos tengan mayor ingreso \\
& - Sí, porque vamos a aprender más sobre la comercialización de los cultivos \\
& - los cuales necesitan mercados justos \\
\hline $\begin{array}{c}\text { Sistemas Agroalimentarios } \\
\text { Localizados }\end{array}$ & $\begin{array}{l}\text { - Sí, porque mi comunidad está en el proceso de fortalecer la producción y } \\
\text { comercialización de los productos del agro } \\
\text { beneficio de las comunidades }\end{array}$ \\
\hline
\end{tabular}

Las respuestas de los participantes a la tercera pregunta, sobre quiénes estarían interesados en hacer el curso en su comunidad, se evidenció un listado de actores rurales, los cuales se mencionan a continuación: líderes sociales y comunitarios, alcaldes, representantes de las Juntas de Acción Comunal, docentes de Instituciones Educativas y Universitarios, promotores rurales, productores, agricultores, socios de organizaciones, jóvenes rurales, estudiantes y profesionales, organizaciones, Instituciones Educativas, dinamizadores agroambientales, madres cabeza de familia y amas de casa.

Por último, la cuarta pregunta referente al acceso a internet en las comunidades, en su mayoría respondieron tener acceso a internet de manera regular, en lugares como: oficinas de las asociaciones, casas de familia, datos en el celular, Instituciones Educativas y en puntos Vive Digital. En su minoría, algunos participantes manifestaron no tener acceso a internet, evidenciado que el lugar más cercano se encuentra aproximadamente a una hora, tiempo del trayecto al pueblo más cercano con difícil acceso en vías terrestres.

\subsection{Segundo momento de la herramienta de validación.}

En un segundo momento, individualmente diligenciaron la matriz cuyo objetivo fue validar el contenido del curso MOOC "Construcción colectiva de mercados agroecológicos y solidarios". La metodología consistió en calificar cada uno de los contenidos de aprendizaje abordados en la Tabla 1 , de acuerdo a conversiones de calificación (cara feliz : 2 puntos; cara normal 1 punto; cara triste). Para cada uno de los contenidos de aprendizaje, la calificación se hizo respecto a tres (3) preguntas: 1) ¿Es pertinente a las necesidades del territorio?; 2) ¿Entiendes el vocabulario expresado?; 3) ¿Este conocimiento beneficia a tu comunidad?. Adicionalmente, los participantes podían plantear sugerencias o recomendaciones.

En la matriz de validación, se realizó un promedio en el puntaje para cada una de las secciones calificadas por los participantes, teniendo en cuenta que el puntaje máximo es de 6 puntos. Algunos participantes realizaron algunas observaciones y recomendaciones, las cuales se evidnecian en la tabla 4.

Tabla 3. Matriz de validación del contenido del curso MOOC

\begin{tabular}{|c|c|c|c|c|c|}
\hline \multirow{2}{*}{ Secciones } & \multicolumn{2}{|c|}{ Apreciación del participantes } & \multirow{2}{*}{} \\
\cline { 2 - 5 } & $\begin{array}{c}\text { ¿Es pertinente a } \\
\text { las necesidades } \\
\text { de tu territorio? }\end{array}$ & $\begin{array}{c}\text { ¿Entiendes el } \\
\text { vocabulario } \\
\text { expresado? }\end{array}$ & $\begin{array}{c}\text { ¿Este } \\
\text { conocimiento } \\
\text { beneficia a la } \\
\text { comunidad? }\end{array}$ & Puntaje & $\begin{array}{c}\text { Sugerencias o } \\
\text { recomendaciones }\end{array}$ \\
\hline $\begin{array}{c}\text { Sección 1: } \\
\text { Introducción- } \\
\text { Ambientación }\end{array}$ & 1 & & 5,9 & $\begin{array}{l}{ }^{1} \text { No hay claridad en los } \\
\text { conceptos agroecológicos y } \\
\text { solidarios }\end{array}$ \\
\hline $\begin{array}{c}\text { Sección 2: } \\
\text { Conceptos y }\end{array}$ & 2 & 5,8 & $\begin{array}{l}{ }^{2} \text { No hay claridad sobre los } \\
\text { conceptos de agroecología, } \\
\text { soberanía alimentaria } \\
\text { ycircuitos cortos de } \\
\text { comercialización }\end{array}$ \\
\hline
\end{tabular}




\begin{tabular}{|c|l|l|l|l|}
\hline $\begin{array}{c}\text { Sección 3: } \\
\text { Logística y } \\
\text { organización } \\
\text { comunitaria }\end{array}$ & & & 6 & \\
\hline $\begin{array}{c}\text { Sección 4: } \\
\text { Prácticas de } \\
\text { intercambio } \\
\text { comercial y } \\
\text { solidario }\end{array}$ & 3 & 5,9 & $\begin{array}{l}\text { 'se debe profundizar en el } \\
\text { tema de huerta urbana; } \\
\text { incorporar el tema de } \\
\text { de mercadeo y estrategias }\end{array}$ \\
\hline
\end{tabular}

\section{$\Theta=0 \quad \Theta=1 \quad \Theta=2$}

Nombre del participante:

Nombre de la organización/Institución Educativa:

Municipio:

Número de identificación:

Edad:

\section{Conclusiones}

Se evidenció claramente que el curso MOOC "Construcción colectivademercadosagroecológicos y solidarios" es pertinente al contexto rural en el que se desempeñan los jóvenes rurales, quienes a través de las herramientas tecnológicas brindadas, los productores provenientes de la agricultura familiar y actores del contexto rural, podrán recorrer caminos distintos hacia la comercialización basada en la venta directa de productos locales, frescos, auténticos, sanos y de temporada, bajo la premisa de la creciente demanda por parte de los consumidores.

Se cumplió con el objetivo general, que estuvo enfocado en evidenciar como estrategia para fortalecer las sinergias comunitarias en las zonas rurales, el diseño del curso MOOC "Construcción colectiva de mercados agroecológicos y solidarios", utilizando la herramienta MOOC Canvas para el diseño del curso, y su validación a través de una herramienta metodológica de InvestigaciónAcción-Participativa (IAP).

La validación del contenido del curso con jóvenes rurales, evidenció las necesidades de las comunidades rurales en fortalecer sus capacidades organizativas y formativas rurales, en aprendizajes basados en proyectos productivos y sinergias comunitarias, a través de las tecnologías de la información y la comunicación para la Educación Superior Rural.

\section{REFERENCIAS}

[1]. Alario-Hoyos, C., Perez-Sanagustin, M., Cormier, D., \& Delgado-Kloos, C. (2014). Proposal for a conceptual framework for educators to describe and design MOOCs. Journal of Universal Computer Science, 20(1), 11.

[2]. Alario-Hoyos, C.; Pérez-Sanagustín, M.; Delgado Kloos, C. (2013). El MOOC Canvas: una herramienta para describir y diseñar MOOCs. Madrid: Universidad Carlos III de Madrid.

[3]. Alberich, T. (2008). IAP, redes y mapas sociales: desde la investigación a la intervención social, Portularia, VIII(1), 131151.

[4]. Basagoiti, M., Bru, P. y Lorenzana, C. (2001). IAP (de bolsillo): Investigación Acción Participativa. Madrid: Acsur Las Segovias.

[5]. Ministerio de Educación Nacional de Colombia - MinEducación (2012). Manual para la formulación y ejecución de planes de Educación Rural. Bogotá: Corpoeducación.

[6]. Proyecto MOOCMenTes de la Universidad del Cauca - Unicauca. (2018). Quénes somos? Popayán: Universidad del Cauca. Consultado en septiembre de 2018, de http://www.unicauca.edu.co/ moocmentes/ 